\title{
Effect of extra-virgin olive oil on lipid profile and inflammatory biomarkers in patients undergoing coronary angiography: a randomized, controlled, clinical trial
}

\section{Nafiseh Khandouzi}

Shaheed Beheshti University of Medical Sciences

\author{
Ali Zahedmehr \\ Iran University of Medical Sciences \\ Javad Nasrollahzadeh ( $\nabla$ jnasrollahzadeh@gmail.com ) \\ Shahid Beheshti University of Medical Sciences School of Nutrition and Food Technology
}

\section{Research}

Keywords: extra-virgin olive oil, refined olive oil, lipid profile, inflammatory markers

Posted Date: June 9th, 2020

DOI: https://doi.org/10.21203/rs.3.rs-31446/v1

License: (c) (1) This work is licensed under a Creative Commons Attribution 4.0 International License. Read Full License 


\section{Abstract \\ Background}

Olive oil, rich in mono-unsaturated fatty acid and the main fat in the Mediterranean diet, has long been considered to be favorable for cardiovascular health. The present study was conducted to compare the effects of high polyphenol extra-virgin olive oil (EVOO) with low polyphenol refined olive oil (ROO) on some cardiovascular risk factors in patients undergoing coronary angiography.

\section{Methods}

In a randomized, controlled, parallel-arm, clinical trial, 40 patients (aged $54.83 \pm 7.04$ years) with at least one classic cardiovascular risk factor (hypertension, dyslipidemia, or diabetes) who referred to coronary angiography were randomly allocated to two groups and received $25 \mathrm{~mL}$ EVOO $(n=20)$ or ROO $(n=20)$ daily for 6 weeks. Fasting blood was collected and plasma lipids, malondialdehyde (MDA), C-reactive protein (CRP) as well as, inflammatory cytokines in ex-vivo lipopolysaccharide (LPS)-stimulated whole blood culture were measured at the baseline and after the dietary intervention.

\section{Results}

Baseline characteristics were similar between the two groups. Plasma LDL-cholesterol significantly reduced in EVOO group ( $-9.52 \pm 20.44$ vs $8.68 \pm 18.77 \mathrm{mg} / \mathrm{dL}, p=0.007$ for EVOO and ROO respectively), whereas total cholesterol/HDL-cholesterol had no significant changes. EVOO resulted in significant reduction in plasma CRP $(-0.40 \pm 0.52$ vs $0.007 \pm 0.42 \mathrm{mg} / \mathrm{L}, p=0.01$ for EVOO and ROO respectively) and increased ex-vivo whole blood LPS-stimulated IL-10 production $(12.13 \pm 33.64 \mathrm{vs}-17.47 \pm 49.04 \mathrm{pg} / \mathrm{mL}, p$ $=0.035$ for EVOO and ROO respectively). However, there were no significant differences in LPS-stimulated IL-6 and plasma MDA levels between the two groups.

\section{Conclusions}

Daily consumption of polyphenol-rich EVOO in subjects who have been under medical treatment with riskreducing agents could additionally improve LDL-C and selected inflammatory markers.

\section{Trial registration:}

NCT03796780 at clinicaltrial.gov (12/29/2018)

\section{Background}


Atherosclerotic cardiovascular disease (ASCVD) continues to be a major cause of global morbidity and mortality [1]. Dyslipidemia, particularly elevated low-density lipoprotein cholesterol (LDL-C), is a major modifiable risk factor for ASCVD [2]. In addition, inflammatory responses have significant effects on all stages of atherosclerosis [3]. Several inflammatory biomarkers have been investigated to improve cardiovascular risk prediction and to monitor the disease process. Among the pro-inflammatory biomarkers, C-reactive protein (CRP) and Interleukin-6 (IL-6) are associated with an increased risk of cardiovascular events and coronary artery disease (CAD) progression [4] whereas, interleukin-10 (IL-10) has a potent anti-inflammatory activity via a profound inhibitory effect on the production of proinflammatory cytokines and mediators from macrophages [5].

Olive oil, rich in mono-unsaturated fatty acid and the main fat in the Mediterranean diet, has long been considered to be favorable for cardiovascular health [6]. Extra-Virgin olive oil (EVOO) is extracted mechanically from olives and contains relatively high amounts of phenolic compounds. Refined olive oil (ROO) has the same fatty acid composition as the virgin oil from which it is derived but it loses most of the minority components in the process. EVOO has been reported to contain approximately 36 phenolic compounds, including hydroxytyrosol, oleuropein \& oleocanthal and these compounds play a key role in the health-promoting qualities of the oil [7]. However, ROO may be preferred by some consumers over EVOO, probably because of its milder flavor [8].

Several experimental and clinical studies have investigated the effects of EVOO on cardiovascular risk factors [9]. However, current literature on the effects of EVOO on plasma lipids and inflammatory markers is conflicting, with some studies showing improvements in lipid profile [10-12] or inflammatory markers [13], while in others no significant improvements have been observed in serum lipids [13-15] or inflammatory indices $[14,16,17]$. Furthermore, few studies have compared the anti-inflammatory and lipid-lowering effects of EVOO and ROO in subjects with cardiovascular risk factors $[18,19]$. The current randomized, controlled, clinical trial was designed to compare the effects of EVOO and ROO consumption in patients with cardiovascular risk factors who were referred to coronary angiography. Two previous studies by Fito et al. have compared daily consumption of $50 \mathrm{~mL} /$ day EVOO and ROO in stable patients with ASCVD studies $[18,19]$. However, this amount may be higher than the usual amount that can be consumed in low-fat diets of patients with cardiovascular disease. In addition, the normal consumption of olive oil may be as much as $25-35 \mathrm{~g}$ /day in the Mediterranean area [20,21]. Therefore, in the present study the effects of consumption of $25 \mathrm{~g}$ /day EVOO were compared to ROO on lipid and inflammatory markers.

\section{Methods}

\section{Subjects}

The population of the study consisted of subjects who referred to coronary angiography in the Shahid Rajaei Cardiovascular, Medical \& Research Center in Tehran. Eligible participants were men and postmenopausal women less than 75 years who had at least one of the major cardiovascular risk factors 
including hypertension, diabetes mellitus, dyslipidemia or acute cardiac events. None of the subjects had taken anti-inflammatory medication or dietary antioxidants or omega-3 supplements during the previous month before the study. Exclusion criteria were consumption of less than $80 \%$ of olive oil delivered to the participants, any changes in the disease treatment plan including type or dose of lipid-lowering medications or coronary artery bypass graft (CABG), and gastrointestinal complications such as diarrhea.

The procedures followed in this study were in accordance with the 1964 Helsinki Declaration and the study protocol was approved by the Ethical Committee of National Nutrition \& Food Technology Research Institute, Tehran, Iran (the Ethical No: IR.SBMU.nnftri.Rec.1396.203), and written informed consent were obtained from all patients. This clinical trial was registered at the clinical trial registration center (clinicaltrial.gov) (NCT03796780).

\section{Study design}

The present study was a randomized, controlled, parallel-arm, clinical trial. One month after coronary angiography, eligible participants were randomly assigned to one of the two groups. The randomization was carried out with stratification for BMI (normal and overweight) and statin medication (with or without statin treatment). We used a list of continuous study numbers with random allocation (by computer) to group 1 or 2. Participants were requested to consume a daily amount of $25 \mathrm{~mL}$ of EVOO or ROO as raw with meals for 6 weeks. The fatty acid composition of EVOO and ROO has been shown in Table 1. Olive oils were given to patients in sufficient quantities. To ensure proper olive oil consumption, participants were followed up weekly by telephone. Dietary intake and physical activity levels were monitored throughout the study. Dietary intakes were assessed using a 3-day dietary recall questionnaire (two regular days in the middle of the week and one day at the weekend) at baseline and after 6 weeks. Participants were asked to maintain their habitual lifestyle throughout the study. Anthropometric and biochemical parameters were reassessed at the end of the intervention.

\section{Blood sampling and culture}

Venous blood samples were taken into heparinized bottles after an overnight fast at baseline and after 6week olive oils consumption. The blood samples were centrifuged (4000 rpm for $20 \mathrm{~min}$ ) and the resulting plasma was stored at $-80^{\circ} \mathrm{C}$. Before plasma isolation, $1 \mathrm{~mL}$ of whole blood was diluted with 4 $\mathrm{mL}$ of Dulbecco's Modified Eagle Medium (Sigma-Aldrich, Heidelberg, Germany) supplemented with $2 \mathrm{mM}$ L-glutamine and then were cultured in 12 -well plates at $37^{\circ} \mathrm{C}$ in a humidified incubator with a $5 \% \mathrm{CO} 2$ \& 95\% air atmosphere. Blood culture was stimulated with lipopolysaccharide (LPS) (Sigma-Aldrich, Heidelberg, Germany) in a final concentration of $1 \mu \mathrm{g} / \mathrm{mL}$ for $24 \mathrm{~h}$, when, culture plates were centrifuged (3000 $\mathrm{g}$ for $5 \mathrm{~min}$ ) and the supernatant was removed and frozen at $-80^{\circ} \mathrm{C}$ for the analysis of IL- 6 and IL10 production. Commercially available ELISA kits were used to measure supernatant concentrations of IL6 and IL-10 (BioLegend, USA). CRP was measured using a turbidometric immunoassay kit by an automated analyzer (Selectra 2, Vital Scientific, Spankeren, The Netherlands). Plasma MDA was analyzed using thiobarbituric acid (TBA) based colorimetric method according to the instruction [22]. Plasma total cholesterol (TC), LDL-C, high-density lipoprotein cholesterol (HDL-C), and triglycerides (TG) were 
measured by automated standardized enzymatic methods using commercial kits. Small dense-LDL-C (sdLDL-C) was measured by a direct homogenous LDL-C assay in the supernatant that remained after heparin-magnesium precipitation of lipoproteins with density $<1.044 \mathrm{~g} / \mathrm{mL}$ [23].

\section{Statistics}

The sample size calculation was performed using G*Power 3.1 software [24]. The primary outcome measure was mean change in IL-6 concentrations [19]. For a significance level of $a=0.05$, an independent t-test indicated that enrolment of 18 subjects will provide $80 \%$ power to detect a $15 \%$ decrease in IL-6, which was increased to $n=20 /$ group to account for a $10 \%$ attrition rate.

The data were analyzed using the SPSS software for Windows versions 21 (SPSS Inc., Chicago, IL, USA). Data are given as Mean \pm SD unless otherwise indicated. The normality of distributions of the study variables was tested with the Shapiro-Wilk test with non-normally distributed data normalized via log transformation where possible to permit parametric testing. Differences between groups were assessed over absolute change values $(\Delta)$ ( 6 weeks minus baseline) by unpaired t-test. Within-group differences between baseline and post-intervention data were analyzed by the paired t-test. The statistical significance level was set at $p=0.05$ (two tails).

\section{Results}

\section{Subjects and dietary assessment}

The final study population consisted of 40 subjects; 20 subjects in the ROO group, and 20 subjects in the EVOO group (Figure 1). The characteristics of the study population are listed in Table 2. No significant differences were observed in the baseline characteristics between the two groups. There were no significant differences between the EVOO and ROO groups regarding the body weight and physical activity at the baseline and 6 weeks (Table 3 ).

As shown in Table 4, food intake analyses showed no differences in daily dietary intake between the two groups at the baseline. As was expected, the dietary intake of total fat and monounsaturated fat of both groups have increased during the experiment due to the consumption of olive oils. However, there were no differences between the two groups. Energy intake in both groups had a slight and nonsignificant increase compared to baseline.

\section{Lipids and Inflammatory markers}

EVOO consumption resulted in lower concentrations of TC $(6 \%)(p=0.007$, statistical power $=0.81)$ and LDL-C $(6 \%)(p=0.011$, statistical power $=0.75)$, whereas it did not influence the sd-LDL-C, HDL-C, TC/HDL-C, LDL-C/HDL-C, and TG when compared with ROO treatment (Table 5). In addition, the plasma MDA level did not differ between the two groups, although within-group analysis showed a significant decrease in the EVOO group. MDA values were not normally distributed and log- 
transformed values were used for the statistical analysis. However, for clarity of the presentation, untransformed values are shown in the table.

None of the participants showed symptoms of acute inflammation during the test days. During 6 weeks of EVOO consumption, the mean change of CRP concentration was $24 \%$ lower in EVOO compared with ROO $(p=0.01$, statistical power $=0.76)$ (Table 5). LPS-stimulated IL-6 concentration had no significant difference between the two groups, whereas a higher level of LPS-stimulated IL-10 was observed in the EVOO group $(p=0.035$, statistical power $=1.00)($ Figure 2$)$.

\section{Discussion}

In this study, we examined the effects of olive oils with low (ROO) or high (EVOO) phenolic content on plasma lipid profile and ex-vivo LPS-stimulated whole blood culture inflammatory cytokines. EVOO consumption exerted a mild LDL-C lowering effect, while it did not reduce sd-LDL-C and TC/HDL-C levels. EVOO also had an attenuating effect on plasma CRP and increased ex-vivo production of LPS-stimulated IL-10 in whole blood culture.

Both LDL-C concentration and size are directly associated with the risk of cardiovascular disease $[25,26]$. Although there are several studies in which polyphenol consumption has not affected LDL-C concentration $[13,14]$, in some studies, olive polyphenols have reduced LDL-C concentration. Hernáez et al. showed that consumption of polyphenol-rich olive oil $(366 \mathrm{mg} / \mathrm{kg})$ in the amount of $25 \mathrm{~mL} /$ day for 3 weeks reduces serum LDL-C levels when compared to low-polyphenol olive oil $(2.7 \mathrm{mg} / \mathrm{kg})$ [27]. In another similar study, Fernández-Castillejo and coworkers showed that consumption ( $25 \mathrm{~mL} /$ day) of polyphenol-enriched olive oil (containing $500 \mathrm{mg}$ polyphenol/kg) have LDL-C lowering effect in hypercholesterolemic individuals, whereas, they did not find any decrease in serum sd-LDL-C particles [10]. EVOO has also been shown to have a blunting effect on postprandial LDL-C elevation in an acute meal consumption study [28]. The mechanisms underlying the LDL-C lowering effects of olive polyphenols are not well defined. However, animal data suggest that it may be mediated through increases in bile flow and biliary cholesterol and bile acid concentrations, leading to increased fecal excretion [29]. In the present study, although EVOO consumption was associated with the reduction in LDL-C levels, the plasma sd-LDL-C, and TC/HDL-C did not improve. TC/HDL-C has been suggested to be a powerful and more accurate predictor of future cardiovascular disease risk and is associated with sdLDL-C particles [30]. Therefore, except for a slight effect on LDL-C, olive polyphenols do not appear to have a significant effect on the improvement of plasma lipid profile.

Immune-modulatory and anti-inflammatory effects of EVOO bioactive minor components, in particular, hydroxytyrosol [31,32] and also the entire phenolic fraction [33] have been investigated through in vitro models. Phenolic compounds present in EVOO have been reported to possess anti-inflammatory actions in animal models of inflammation [34,35]. In our patients, reduced plasma levels of CRP were observed after EVOO ingestion. The reduction of circulating CRP after EVOO compared to ROO intake has been reported in a previous study in which patients with coronary artery disease took a daily amount of $50 \mathrm{~mL}$ 
of EVOO and ROO sequentially over two 3-week periods [19]. However, in another study on diabetic subjects, the consumption of EVOO ( $25 \mathrm{~mL} /$ day, $577 \mathrm{mg}$ of phenolic compounds $/ \mathrm{kg}$ ) did not significantly alter the levels of circulating hs-CRP compared to refined olive oil [14].

Production of cytokines in humans may be studied in the circulation and ex vivo by stimulation of isolated cells. Circulating levels of cytokines may not necessarily reflect local cytokine production. Yet the isolation of cells is cumbersome and when isolated cells are cultured, they deprived of adjacent cells and serum components which may cause them to lose their in vivo characteristics. Cytokine production from whole-blood culture could be a valid and convenient assay for the measurement of cytokine production, particularly when stimulated with LPS [36]. In the present study, despite the improved plasma CRP after EVOO ingestion, we found no changes in the ex-vivo production of LPS-stimulated IL-6. On the contrary, EVOO consumption produced a significant increase in LPS-stimulated IL-10. IL-10 is an important immunoregulatory cytokine with pleiotropic effects that appears to play a role in the function of regulatory $T$ cells, which are recognized as potent suppressors of excessive immune responses [37]. Administration of olive polyphenols containing mostly hydroxytyrosol has been associated with increased IL-10 levels in an animal model of paw inflammation induced by injecting carrageenan [38]. Our finding is consistent with the results of Aparicio-Soto et al., which reported evidence of increased IL10 production when olive phenolic compounds were added to the ex-vivo culture of peripheral blood mononuclear cells (PBMC) from healthy subjects [39]. Furthermore, co-stimulation of human PBMCs with hydroxytyrosol and an allergen has been associated with an increase in the amount of IL-10 production [40]. Moreover, acute ingestion of $50 \mathrm{ml}$ EVOO has been reported to increase the IL-10 gene expression in PBMCs from healthy subjects [41].

The anti-inflammatory effects of olive bioactive minor compounds could be attributed to their capacity to reduce the reactive oxygen species generation [42-44]. However, in the current study, plasma MDA levels did not differ between the two treatments, although within-group analysis showed a significant decrease in EVOO treatment. In contrast to our findings, the antioxidant effects of olive oil polyphenols have been demonstrated in previous studies. In a crossover study in healthy participants, daily administration of 25 $\mathrm{mL}$ of olive oils (with low, medium, and high phenolic content) for 3 weeks, linearly decreased oxidative stress markers (including oxidized-LDL-C) with increasing phenolic content [45]. One of the reasons for the lack of effect of EVOO on MDA in the present study may be related to the detection method of MDA (colorimetric TBA-based method) which has been claimed to be insufficiently sensitive [46].

There were some limitations to this study. The sample sizes in each group were rather small and the power to detect subtle changes is therefore restricted. Furthermore, the study would have benefited if continued for longer durations to assess maintenance. Moreover, cross-over design may have been a better choice for reducing the effects of confounding factors. However, since seasonal variations influence inflammatory biomarkers $[47,48]$, we decided to choose a parallel design. Moreover, we assessed a limited number of parameters related to inflammation.

\section{Conclusions}


Consumption of polyphenol-rich olive oil in comparison with refined olive oil slightly reduces LDL-C but does not affect other lipoprotein components. However, it may have some anti-inflammatory effects. Therefore, given these benefits, consumption of polyphenol-enriched olive oil could be a way of increasing its healthy properties while the same amount of fat is consumed.

\section{Abbreviations}

ASCVD

Atherosclerotic cardiovascular disease

CAD

coronary artery disease

CRP

C-reactive protein

EVOO

extra-virgin olive oil

HDL-C

high-density lipoprotein cholesterol

IL-6

Interleukin-6

IL-10

Interleukin-10

LPS

lipopolysaccharide

LDL-C

low-density lipoprotein cholesterol

MDA

malondialdehyde

PBMC

peripheral blood mononuclear cells

ROO

refined olive oil

TBA

thiobarbituric acid

TC

total cholesterol

TG

triglycerides

\section{Declarations}




\section{- Ethics approval and consent to participate}

The procedures followed in this study were in accordance with the 1964 Helsinki Declaration and the study protocol was approved by the Ethical Committee of National Nutrition \& Food Technology Research Institute, Tehran, Iran (the Ethical No: IR.SBMU.nnftri.Rec.1396.203), and written informed consent were obtained from all patients.

\section{- Consent for publication}

Not Applicable.

\section{- Availability of data and materials}

All data generated or analysed during this study are included in this published article [and its supplementary information files].

\section{- Competing interests}

The authors declare that they have no competing interests.

\section{- Funding}

The study was supported by a grant from National Nutrition, and Food Technology Research Institute, Shahid Beheshti University of Medical Sciences, Tehran, Iran, grant number No. 795.

\section{- Authors' contributions}

NK was responsible for designing the research protocol, writing the protocol, conducting the research, screening potentially eligible studies, conducting biochemical tests, extracting and analyzing data, interpreting results, and writing the paper.

$\mathrm{JN}$ was responsible for designing the research protocol and screening of potentially eligible studies. He contributed to extracting and analyzing data, interpreting results, and writing the paper.

AZ assisted in conducting the research, especially in medical consultation \& material support, and study supervision.

\section{- Acknowledgements}

The study was supported by a grant from National Nutrition, and Food Technology Research Institute, Shahid Beheshti University of Medical Sciences, Tehran, Iran. The authors thank the personnel of Rajaie Cardiovascular, Medical \& Research Center. We are also grateful to all participants in the study.

\section{References}


1. Collaborators GBDCoD. Global, regional, and national age-sex specific mortality for 264 causes of death, 1980-2016: a systematic analysis for the Global Burden of Disease Study 2016. Lancet. 2017;390:1151-210.

2. Baigent C, Blackwell L, Emberson J, Holland LE, Reith C, Bhala N, Peto R, Barnes EH, Keech A, Simes $\mathrm{J}$, Collins R. Efficacy and safety of more intensive lowering of LDL cholesterol: a meta-analysis of data from 170,000 participants in 26 randomised trials. Lancet. 2010;376:1670-81.

3. Galkina E, Ley K. Immune and inflammatory mechanisms of atherosclerosis $\left(^{*}\right)$. Annu Rev Immunol. 2009;27:165-97.

4. Zakynthinos E, Pappa N. Inflammatory biomarkers in coronary artery disease. J Cardiol. 2009;53:317-33.

5. Mosser DM, Zhang X. Interleukin-10: new perspectives on an old cytokine. Immunol Rev. 2008;226:205-18.

6. Foscolou A, Critselis E, Panagiotakos D. Olive oil consumption and human health: A narrative review. Maturitas. 2018;118:60-6.

7. Parkinson L, Cicerale S. The Health Benefiting Mechanisms of Virgin Olive Oil Phenolic Compounds. Molecules 2016, 21.

8. Vazquez-Araujo L, Adhikari K, Chambers Et, Chambers DH, Carbonell-Barrachina AA. Cross-cultural perception of six commercial olive oils: A study with Spanish and US consumers. Food Sci Technol Int. 2015;21:454-66.

9. Romani A, leri F, Urciuoli S, Noce A, Marrone G, Nediani C, Bernini R. Health Effects of Phenolic Compounds Found in Extra-Virgin Olive Oil, By-Products, and Leaf of Olea europaea L. Nutrients. 2019;11:1776.

10. Fernandez-Castillejo S, Valls RM, Castaner O, Rubio L, Catalan U, Pedret A, Macia A, Sampson ML, Covas MI, Fito M, et al. Polyphenol rich olive oils improve lipoprotein particle atherogenic ratios and subclasses profile: A randomized, crossover, controlled trial. Mol Nutr Food Res. 2016;60:1544-54.

11. Haban P, Klvanova J, Zidekova E, Nagyova A. Dietary supplementation with olive oil leads to improved lipoprotein spectrum and lower n-6 PUFAs in elderly subjects. Med Sci Monit. 2004;10:Pi49-54.

12. Castaner O, Covas MI, Khymenets $\mathrm{O}$, Nyyssonen K, Konstantinidou V, Zunft HF, de la Torre R, MunozAguayo D, Vila J, Fito M. Protection of LDL from oxidation by olive oil polyphenols is associated with a downregulation of CD40-ligand expression and its downstream products in vivo in humans. Am J Clin Nutr. 2012;95:1238-44.

13. Fito M, Cladellas M, de la Torre R, Marti J, Munoz D, Schroder H, Alcantara M, Pujadas-Bastardes M, Marrugat J, Lopez-Sabater MC, et al. Anti-inflammatory effect of virgin olive oil in stable coronary disease patients: a randomized, crossover, controlled trial. Eur J Clin Nutr. 2008;62:570-4.

14. Santangelo C, Filesi C, Vari R, Scazzocchio B, Filardi T, Fogliano V, D'Archivio M, Giovannini C, Lenzi A, Morano S, Masella R. Consumption of extra-virgin olive oil rich in phenolic compounds improves 
metabolic control in patients with type 2 diabetes mellitus: a possible involvement of reduced levels of circulating visfatin. J Endocrinol Invest. 2016;39:1295-301.

15. D'Amore S, Vacca M, Cariello M, Graziano G, D'Orazio A, Salvia R, Sasso RC, Sabba C, Palasciano G, Moschetta A. Genes and miRNA expression signatures in peripheral blood mononuclear cells in healthy subjects and patients with metabolic syndrome after acute intake of extra virgin olive oil. Biochim Biophys Acta. 2016;1861:1671-80.

16. Rozati M, Barnett J, Wu D, Handelman G, Saltzman E, Wilson T, Li L, Wang J, Marcos A, Ordovas JM, et al. Cardio-metabolic and immunological impacts of extra virgin olive oil consumption in overweight and obese older adults: a randomized controlled trial. Nutr Metab (Lond). 2015;12:28.

17. Kozic Dokmanovic S, Kolovrat K, Laskaj R, Jukic V, Vrkic N, Begovac J. Effect of Extra Virgin Olive Oil on Biomarkers of Inflammation in HIV-Infected Patients: A Randomized, Crossover, Controlled Clinical Trial. Med Sci Monit. 2015;21:2406-13.

18. Fito $\mathrm{M}$, Cladellas $\mathrm{M}$, de la Torre $\mathrm{R}$, Marti J, Alcantara M, Pujadas-Bastardes $\mathrm{M}$, Marrugat J, Bruguera J, Lopez-Sabater MC, Vila J, et al. Antioxidant effect of virgin olive oil in patients with stable coronary heart disease: a randomized, crossover, controlled, clinical trial. Atherosclerosis. 2005;181:149-58.

19. Fito M, Cladellas M, de la Torre R, Marti J, Munoz D, Schroder H, Alcantara M, Pujadas-Bastardes M, Marrugat J, Lopez-Sabater MC, et al. Anti-inflammatory effect of virgin olive oil in stable coronary disease patients: a randomized, crossover, controlled trial. Eur J Clin Nutr. 2008;62:570-4.

20. Di Daniele N, Petramala L, Di Renzo L, Sarlo F, Della Rocca DG, Rizzo M, Fondacaro V, lacopino L, Pepine CJ, De Lorenzo A. Body composition changes and cardiometabolic benefits of a balanced Italian Mediterranean Diet in obese patients with metabolic syndrome. Acta Diabetol. 2013;50:40916.

21. Sidahmed E, Cornellier ML, Ren J, Askew LM, Li Y, Talaat N, Rapai MS, Ruffin MT, Turgeon DK, Brenner D, et al. Development of exchange lists for Mediterranean and Healthy Eating diets: implementation in an intervention trial. J Hum Nutr Diet. 2014;27:413-25.

22. Satoh K. Serum lipid peroxide in cerebrovascular disorders determined by a new colorimetric method. Clin Chim Acta. 1978;90:37-43.

23. Hirano T, Ito $Y$, Saegusa H, Yoshino G. A novel and simple method for quantification of small, dense LDL. J Lipid Res. 2003;44:2193-201.

24. Faul F, Erdfelder E, Lang AG, Buchner A. G*Power 3: a flexible statistical power analysis program for the social, behavioral, and biomedical sciences. Behav Res Methods. 2007;39:175-91.

25. Ueda P, Gulayin P, Danaei G. Long-term moderately elevated LDL-cholesterol and blood pressure and risk of coronary heart disease. PLoS One. 2018;13:e0200017.

26. Pichler G, Amigo N, Tellez-Plaza M, Pardo-Cea MA, Dominguez-Lucas A, Marrachelli VG, Monleon D, Martin-Escudero JC, Ascaso JF, Chaves FJ, et al. LDL particle size and composition and incident cardiovascular disease in a South-European population: The Hortega-Liposcale Follow-up Study. Int J Cardiol. 2018;264:172-8. 
27. Hernaez A, Fernandez-Castillejo S, Farras M, Catalan U, Subirana I, Montes R, Sola R, Munoz-Aguayo D, Gelabert-Gorgues A, Diaz-Gil O, et al. Olive oil polyphenols enhance high-density lipoprotein function in humans: a randomized controlled trial. Arterioscler Thromb Vasc Biol. 2014;34:2115-9.

28. Violi F, Loffredo L, Pignatelli P, Angelico F, Bartimoccia S, Nocella C, Cangemi R, Petruccioli A, Monticolo R, Pastori D, Carnevale R. Extra virgin olive oil use is associated with improved postprandial blood glucose and LDL cholesterol in healthy subjects. Nutr Diabetes. 2015;5:e172.

29. Krzeminski R, Gorinstein S, Leontowicz H, Leontowicz M, Gralak M, Czerwinski J, Lojek A, Ciz M, Martin-Belloso O, Gligelmo-Miguel N, Trakhtenberg S. Effect of different olive oils on bile excretion in rats fed cholesterol-containing and cholesterol-free diets. J Agric Food Chem. 2003;51:5774-9.

30. Lemieux I, Lamarche B, Couillard C, Pascot A, Cantin B, Bergeron J, Dagenais GR, Despres JP. Total cholesterol/HDL cholesterol ratio vs LDL cholesterol/HDL cholesterol ratio as indices of ischemic heart disease risk in men: the Quebec Cardiovascular Study. Arch Intern Med. 2001;161:2685-92.

31. Richard N, Arnold S, Hoeller U, Kilpert C, Wertz K, Schwager J. Hydroxytyrosol is the major antiinflammatory compound in aqueous olive extracts and impairs cytokine and chemokine production in macrophages. Planta Med. 2011;77:1890-7.

32. Scoditti E, Nestola A, Massaro M, Calabriso N, Storelli C, De Caterina R, Carluccio MA. Hydroxytyrosol suppresses MMP-9 and COX-2 activity and expression in activated human monocytes via PKCalpha and PKCbeta1 inhibition. Atherosclerosis. 2014;232:17-24.

33. Cardeno A, Sanchez-Hidalgo M, Aparicio-Soto M, Sanchez-Fidalgo S, Alarcon-de-la-Lastra C. Extra virgin olive oil polyphenolic extracts downregulate inflammatory responses in LPS-activated murine peritoneal macrophages suppressing NFkappaB and MAPK signalling pathways. Food Funct. 2014;5:1270-7.

34. Gong D, Geng C, Jiang L, Cao J, Yoshimura H, Zhong L. Effects of hydroxytyrosol-20 on carrageenaninduced acute inflammation and hyperalgesia in rats. Phytother Res. 2009;23:646-50.

35. Sanchez-Fidalgo S, Villegas I, Aparicio-Soto M, Cardeno A, Rosillo MA, Gonzalez-Benjumea A, Marset A, Lopez O, Maya I, Fernandez-Bolanos JG. Alarcon de la Lastra C: Effects of dietary virgin olive oil polyphenols: hydroxytyrosyl acetate and 3, 4-dihydroxyphenylglycol on DSS-induced acute colitis in mice. J Nutr Biochem. 2015;26:513-20.

36. Damsgaard CT, Lauritzen L, Calder PC, Kjaer TM, Frokiaer H. Whole-blood culture is a valid low-cost method to measure monocytic cytokines - a comparison of cytokine production in cultures of human whole-blood, mononuclear cells and monocytes. J Immunol Methods. 2009;340:95-101.

37. Okamoto A, Fujio K, Okamura T, Yamamoto K. Regulatory T-cell-associated cytokines in systemic lupus erythematosus. J Biomed Biotechnol. 2011;2011:463412.

38. Carito V, Ciafre S, Tarani L, Ceccanti M, Natella F, lannitelli A, Tirassa P, Chaldakov GN, Ceccanti M, Boccardo C, Fiore M. TNF-alpha and IL-10 modulation induced by polyphenols extracted by olive pomace in a mouse model of paw inflammation. Ann Ist Super Sanita. 2015;51:382-6.

39. Aparicio-Soto M, Sanchez-Hidalgo M, Cardeno A, Lucena JM, Gonzalez-Escribano F, Castillo MJ, Alarcon-de-la-Lastra C. The phenolic fraction of extra virgin olive oil modulates the activation and the 
inflammatory response of T cells from patients with systemic lupus erythematosus and healthy donors. Mol Nutr Food Res 2017, 61.

40. Bonura A, Vlah S, Longo A, Bulati M, Melis MR, Cibella F, Colombo P. Hydroxytyrosol modulates Par j 1-induced IL-10 production by PBMCs in healthy subjects. Immunobiology. 2016;221:1374-7.

41. Konstantinidou V, Khymenets O, Fito M, De La Torre R, Anglada R, Dopazo A, Covas MI. Characterization of human gene expression changes after olive oil ingestion: an exploratory approach. Folia Biol (Praha). 2009;55:85-91.

42. Serra G, Deiana M, Spencer JPE, Corona G. Olive Oil Phenolics Prevent Oxysterol-Induced Proinflammatory Cytokine Secretion and Reactive Oxygen Species Production in Human Peripheral Blood Mononuclear Cells, Through Modulation of p38 and JNK Pathways. Mol Nutr Food Res 2017, 61.

43. Muriana FJG, Montserrat-de la Paz S. Tyrosol and its metabolites as antioxidative and antiinflammatory molecules in human endothelial cells. 2017, 8:2905-2914.

44. Cicerale S, Lucas L, Keast R. Biological activities of phenolic compounds present in virgin olive oil. Int J Mol Sci. 2010;11:458-79.

45. Covas MI, Nyyssonen K, Poulsen HE, Kaikkonen J, Zunft HJ, Kiesewetter H, Gaddi A, de la Torre R, Mursu J, Baumler $\mathrm{H}$, et al. The effect of polyphenols in olive oil on heart disease risk factors: a randomized trial. Ann Intern Med. 2006;145:333-41.

46. Del Rio D, Stewart AJ, Pellegrini N. A review of recent studies on malondialdehyde as toxic molecule and biological marker of oxidative stress. Nutr Metab Cardiovasc Dis. 2005;15:316-28.

47. Liu B, Taioli E. Seasonal Variations of Complete Blood Count and Inflammatory Biomarkers in the US Population - Analysis of NHANES Data. PLoS One. 2015;10:e0142382.

48. Al Hanai AH, Antkiewicz DS, Hemming JDC, Shafer MM, Lai AM, Arhami M, Hosseini V, Schauer JJ. Seasonal variations in the oxidative stress and inflammatory potential of PM2.5 in Tehran using an alveolar macrophage model; The role of chemical composition and sources. Environ Int. 2019;123:417-27.

\section{Tables}

Table 1. Chemical composition of Olive Oils 


\begin{tabular}{|l|c|c|}
\hline Chemical Component & Extra-Virgin Olive Oil & Refined Olive Oil \\
\hline Total Phenolic Content (ppm) & $500<$ & $10>$ \\
\hline Fatty Acid (per 100 gr): & & \\
\hline C14:0 & 0.04 & 0.04 \\
\hline C16:0 & 14.65 & 15.78 \\
\hline C16:1 & 1.36 & 0.78 \\
\hline C17:0 & 0.10 & 0.14 \\
\hline C17:1 & 0.12 & 0.16 \\
\hline C18:0 & 1.32 & 1.58 \\
\hline C18:1 cis n-9 & 69.58 & 65.70 \\
\hline C18:1 trans & 0.02 & 0.03 \\
\hline C18:2 & 9.42 & 11.23 \\
\hline C18:3 & 0.39 & 0.48 \\
\hline C18: trans + C18:3 trans & $<0.01$ & 0.04 \\
\hline C20:0 & 0.13 & 0.24 \\
\hline C20:1 & $<0.01$ & $<0.01$ \\
\hline C22:0 & ND & $<0.01$ \\
\hline C24:0 & 0.03 & 0.08 \\
\hline
\end{tabular}

ND: Not Determined.

Table 2. Baseline characteristics of the participants ${ }^{\text {a }}$ 


\begin{tabular}{cccc}
\hline Variable & Extra-Virgin Olive Oil (n=20) & $\begin{array}{c}\text { Refined Olive Oil } \\
(\mathbf{n = 2 0})\end{array}$ & p-value $^{\mathrm{b}}$ \\
\hline Age (year) & $53.60 \pm 7.64$ & $56.05 \pm 6.33$ & 0.27 \\
Sex (Male) & $18(90 \%)$ & $20(100 \%)$ & 0.48 \\
PCI history & $16(80 \%)$ & $16(80 \%)$ & 1.00 \\
CABG history & $0(0 \%)$ & $2(10 \%)$ & 0.48 \\
Diabetes Mellitus & $5(25 \%)$ & $8(40 \%)$ & 0.50 \\
Hypertension & $9(45 \%)$ & $11(55 \%)$ & 0.75 \\
Dyslipidemia & $8(40 \%)$ & $11(55 \%)$ & 0.52 \\
Overweight & $16(80 \%)$ & $15(75 \%)$ & 1.00 \\
Smoking & $2(10 \%)$ & $8(40 \%)$ & 0.06 \\
Medications: & & & \\
Aspirin & $17(85 \%)$ & $20(100 \%)$ & 0.23 \\
Clopidogrel (Plavix) & $15(75 \%)$ & $15(75 \%)$ & 1.00 \\
Statins & $19(95 \%)$ & $19(95 \%)$ & 1.00 \\
ACEI & $6(30 \%)$ & $9(45 \%)$ & 0.51 \\
ARB & $11(55 \%)$ & $10(50 \%)$ & 1.00 \\
BB & $14(70 \%)$ & $15(75 \%)$ & 1.00 \\
CCB & $3(15 \%)$ & $0(0 \%)$ & 0.23 \\
Nitrates & $4(20 \%)$ & $7(35 \%)$ & 0.48 \\
Aldosteron Antagonist & $3(15 \%)$ & $6(30 \%)$ & 0.45 \\
Loop Diuretics & $2(10 \%)$ & $7(35 \%)$ & 0.13 \\
\hline
\end{tabular}

${ }^{\mathrm{a}}$ Data presented as Mean \pm SD or number (percentage).

b Data were compared using Independent t-test or Chi-square test.

PCI: Percutaneous Coronary Intervention; CABG: Coronary Artery Bypass Graft; ACEI: Angiotensin Converting Enzyme Inhibitor; ARB: Angiotensin Receptor Blocker; BB: Beta-Blocker; CCB: Calcium Channel Blocker

Table 3. Body weight and physical activity of the participants at the baseline and after the intervention according to experimental groups ${ }^{\text {a }}$ 


\begin{tabular}{ccccc}
\hline Variable & Extra-Virgin Olive Oil (n=20) & Refined Olive Oil (n=20) & p-value ${ }^{\mathbf{b}}$ \\
\hline $\begin{array}{c}\text { Body Weight }(\mathbf{k g}) \\
\text { Baseline }\end{array}$ & $78.90(74.31$ to 83.49$)$ & $82.30(77.78$ to 86.81$)$ & \\
6-Week & $78.55(74.16$ to 82.93$)$ & $82.20(77.63$ to 86.76$)$ & \\
\hline $\boldsymbol{\Delta}$ values & $-0.35(-1.03$ to 0.83$)$ & $-0.10(-0.92$ to 0.22$)$ & 0.63 \\
\hline BMI $\left(\mathbf{k g} / \mathbf{m}^{2}\right)$ & & & & \\
Baseline & $27.42(26.01$ to 28.83$)$ & $27.11(25.82$ to 28.39$)$ & \\
\hline 6-Week & $27.32(25.92$ to 28.72$)$ & $27.10(25.81$ to 28.38$)$ & \\
\hline $\boldsymbol{\Delta}$ values & $-0.10(-0.30$ to 0.28$)$ & $-0.01(-0.29$ to 0.09$)$ & 0.59 \\
\hline Physical Activity (MET- & & & & \\
h/day) & & & \\
Baseline & $26.26(24.50$ to 28.01$)$ & $28.29(25.76$ to 30.82$)$ & \\
\hline 6-Week & $26.33(24.53$ to 28.12$)$ & $28.32(25.79$ to 30.84$)$ & \\
\hline $\boldsymbol{\Delta}$ values & $0.07(-0.08$ to 0.13$)$ & $0.02(-0.17$ to 0.31$)$ & 0.73 \\
\hline
\end{tabular}

${ }^{\mathrm{a}}$ Values are Mean (95\% CI).

${ }^{\mathrm{b}}$ Between-group $\Delta$ values ( 6 weeks minus baseline) by Independent t-test.

BMI: Body Mass Index; MET: Metabolic Equivalent.

Table 4. Dietary intake of the participants at the baseline and after the intervention ${ }^{\text {a }}$ 


\section{Groups b}

\begin{tabular}{|c|c|c|c|c|}
\hline Variable & Time & $\begin{array}{l}\text { Extra-Virgin Olive Oil group } \\
(\mathrm{n}=20)\end{array}$ & $\begin{array}{l}\text { Refined Olive Oil group } \\
\qquad(n=20)\end{array}$ & p-value ${ }^{c}$ \\
\hline \multirow{2}{*}{ Energy (kcal) } & Baseline & $1738.30 \pm 190.22$ & $1797.40 \pm 197.10$ & 0.34 \\
\hline & 6-week & $1818.55 \pm 189.13$ & $1850.55 \pm 143.30$ & 0.55 \\
\hline \multirow{2}{*}{$\begin{array}{c}\text { Carbohydrate } \\
\text { (gr) }\end{array}$} & Baseline & $241.40 \pm 37.12$ & $233.05 \pm 32.18$ & 0.45 \\
\hline & 6-week & $246.15 \pm 36.56$ & $234.25 \pm 33.57$ & 0.29 \\
\hline \multirow[t]{2}{*}{ Carbohydrate (\%) } & Baseline & $53.35 \pm 3.76$ & $51.90 \pm 4.60$ & 0.13 \\
\hline & 6-week & $53.95 \pm 3.47$ & $50.47 \pm 4.20$ & 0.14 \\
\hline \multirow[t]{2}{*}{ Protein (gr) } & Baseline & $66.68 \pm 8.09$ & $69.75 \pm 15.55$ & 0.71 \\
\hline & 6-week & $67.47 \pm 8.70$ & $70.65 \pm 11.27$ & 0.66 \\
\hline \multirow[t]{2}{*}{ Protein (\%) } & Baseline & $15.76 \pm 2.25$ & $15.13 \pm 2.18$ & 0.77 \\
\hline & 6-week & $15.22 \pm 2.11$ & $15.29 \pm 2.27$ & 0.92 \\
\hline \multirow[t]{2}{*}{ Total Fat (gr) } & Baseline & $65.15 \pm 8.13$ & $62.95 \pm 10.15$ & 0.12 \\
\hline & 6-week & $71.65 \pm 6.40^{d}$ & $71.40 \pm 7.91 \mathrm{~d}$ & 0.61 \\
\hline \multirow[t]{2}{*}{ Total Fat (\%) } & Baseline & $30.68 \pm 4.03$ & $32.17 \pm 4.53$ & 0.14 \\
\hline & 6-week & $32.65 \pm 3.00^{d}$ & $34.87 \pm 4.36^{\mathrm{d}}$ & 0.10 \\
\hline \multirow[t]{2}{*}{ SFA (gr) } & Baseline & $13.65 \pm 3.51$ & $14.83 \pm 2.93$ & 0.27 \\
\hline & 6-week & $12.85 \pm 2.66$ & $14.42 \pm 2.93$ & 0.18 \\
\hline \multirow[t]{2}{*}{ MUFA (gr) } & Baseline & $20.05 \pm 2.63$ & $21.53 \pm 5.46$ & 0.19 \\
\hline & 6-week & $30.50 \pm 2.94^{d}$ & $32.30 \pm 4.37^{d}$ & 0.10 \\
\hline \multirow[t]{2}{*}{ MUFA (\%) } & Baseline & $10.23 \pm 1.59$ & $10.77 \pm 2.43$ & 0.21 \\
\hline & 6-week & $14.89 \pm 1.36^{\mathrm{d}}$ & $15.79 \pm 2.45^{d}$ & 0.16 \\
\hline \multirow[t]{2}{*}{ PUFA (gr) } & Baseline & $23.85 \pm 5.04$ & $25.89 \pm 5.56$ & 0.23 \\
\hline & 6-week & $23.25 \pm 2.69$ & $23.78 \pm 2.94$ & 0.90 \\
\hline \multirow[t]{2}{*}{ Omega-3 (mg) } & Baseline & $141.94 \pm 108.51$ & $213.30 \pm 178.73$ & 0.15 \\
\hline & 6-week & $156.06 \pm 59.16$ & $233.85 \pm 150.65$ & 0.10 \\
\hline \multirow[t]{2}{*}{ Cholesterol (mg) } & Baseline & $186.61 \pm 54.51$ & $167.65 \pm 82.15$ & 0.45 \\
\hline & 6-week & $167.26 \pm 60.98$ & $144.53 \pm 49.38$ & 0.36 \\
\hline \multirow[t]{2}{*}{ Fiber (gr) } & Baseline & $14.05 \pm 2.52$ & $14.45 \pm 2.32$ & 0.93 \\
\hline & 6-week & $14.40 \pm 2.06$ & $15.90 \pm 2.78$ & 0.88 \\
\hline
\end{tabular}

\footnotetext{
${ }^{\mathrm{a}}$ All values are Mean $\pm \mathrm{SD}$.

$\mathrm{b}$ The daily olive oil consumption is considered.

${ }^{\mathrm{C}}$ Data were compared using Independent t-test.
} 
d Significantly different from Baseline. Data were compared using paired t-test.

SFA: Saturated Fatty Acid; MUFA: Mono-Unsaturated Fatty Acids; PUFA: Poly-Unsaturated Fatty Acids.

Table 5. Biochemical variables at the baseline and after the intervention according to experimental groups ${ }^{a}$ 


\begin{tabular}{|c|c|c|c|}
\hline Variable & Refined Olive Oil $(n=20)$ & Extra-Virgin Olive Oil $(n=20)$ & p-value $b$ \\
\hline \multicolumn{4}{|l|}{$\overline{\mathrm{TC}}(\mathrm{mg} / \mathrm{dL})$} \\
\hline Baseline & $114.42(105.49$ to 132.61$)$ & 158.95 (138.75 to 180.25$)$ & \\
\hline 6-Week & $123.11(105.49$ to 132.61$)$ & 149.42 (110.20 to 136.70$)$ & \\
\hline$\Delta$ values & $8.68(-0.36$ to 17.73$)$ & $-9.52(-2.32$ to -26.52$)$ & 0.007 \\
\hline \multicolumn{4}{|c|}{$\overline{\mathrm{LDL}-\mathrm{C}(\mathrm{mg} / \mathrm{dL})}$} \\
\hline Baseline & 61.47 (55.81 to 73.29 ) & 83.30 (69.41 to 97.19$)$ & \\
\hline 6-Week & 65.84 (57.40 to 73.80$)$ & 78.20 (64.64 to 91.76$)$ & \\
\hline$\Delta$ values & $4.36(-1.26$ to 9.99$)$ & $-5.10(-0.55$ to -10.70$)$ & 0.011 \\
\hline \multicolumn{4}{|c|}{$\overline{\mathrm{l}-\mathrm{LDL}-\mathrm{C}(\mathrm{mg} / \mathrm{dL})}$} \\
\hline Baseline & 25.89 (22.02 to 33.78$)$ & 24.00 (16.12 to 31.88$)$ & \\
\hline 6-Week & 24.89 (20.39 to 29.91$)$ & 22.20 (14.44 to 29.96$)$ & \\
\hline$\Delta$ values & $-1.00(-4.28$ to 2.28$)$ & $-1.80(-6.20$ to 2.41$)$ & 0.75 \\
\hline \multicolumn{4}{|c|}{$\overline{\mathrm{HDL}-\mathrm{C}(\mathrm{mg} / \mathrm{dL})}$} \\
\hline Baseline & 37.20 (33.65 to 40.75$)$ & 43.95 (39.12 to 53.98 ) & \\
\hline 6-Week & 38.80 (35.30 to 42.30 ) & 42.47 (38.86 to 46.74$)$ & \\
\hline$\Delta$ values & $1.60(-0.24$ to 2.45$)$ & $-1.47(-9.96$ to 1.96$)$ & 0.11 \\
\hline \multicolumn{4}{|l|}{ TC/HDL-C } \\
\hline Baseline & $3.22(2.98$ to 3.46$)$ & $3.62(3.06$ to 4.21$)$ & \\
\hline 6-Week & $3.22(2.91$ to 3.53$)$ & $3.52(2.93$ to 4.03$)$ & \\
\hline$\Delta$ values & $0.00(-0.20$ to 0.20$)$ & $-0.09(-0.23$ to 0.54$)$ & 0.42 \\
\hline \multicolumn{4}{|c|}{ LDL-C/HDL-C } \\
\hline Baseline & 1.71 (1.57 to 1.92$)$ & $1.88(1.55$ to 2.21$)$ & \\
\hline 6-Week & $1.73(1.51$ to 1.92$)$ & 1.85 (1.53 to 2.17$)$ & \\
\hline$\Delta$ values & $0.01(-0.11$ to 0.17$)$ & $-0.03(-0.11$ to 0.17$)$ & 0.56 \\
\hline
\end{tabular}

TG (mg/dL)

Baseline $\quad 112.00(100.16$ to 131.14$) \quad 173.50(115.30$ to 231.70$)$

6-Week 121.89 (105.47 to 169.33) 163.05 (100.92 to 225.18)

$\begin{array}{lllll}\Delta \text { values } & 9.89(-9.98 \text { to } 37.35) & -10.45(-34.08 \text { to } 8.18) & 0.13\end{array}$

CRP (mg/L)

$\begin{array}{cccc}\text { Baseline } & 1.70(0.98 \text { to } 2.42) & 1.65(1.01 \text { to } 2.28) & \\ \text { 6-Week } & 1.70(1.00 \text { to } 2.39) & 1.24(0.72 \text { to } 1.76) & \\ \Delta \text { values } & 0.007(-1.46 \text { to } 0.89) & -0.40(-0.14 \text { to }-0.66) & 0.01\end{array}$

MDA ( $\mu \mathrm{mol} / \mathrm{L})$

\begin{tabular}{cccc} 
Baseline & $17.44(13.52$ to 22.54$)$ & $8.75(4.75$ to 12.74$)$ & \\
\hline 6-Week & $14.42(9.29$ to 21.14$)$ & $5.87(13.52$ to 23.54$)$ & \\
\hline $\boldsymbol{\Delta}$ values & $-3.02(-6.47$ to 3.84$)$ & $-2.87(-5.56$ to -0.18$)$ & 0.94 \\
\hline
\end{tabular}


a Values are Mean (95\% CI).

$\mathrm{b}$ Between-group $\Delta$ values (6-week minus baseline) by Independent t-test.

TC: Total Cholesterol; LDL-C: Low-Density Lipoprotein-Cholesterol; sd-LDL-C: Small Dense LDL-C; HDL-C: HighDensity Lipoprotein-Cholesterol; TG: Triglyceride; CRP: C Reactive Protein; MDA: Malondialdehyde.

\section{Figures}

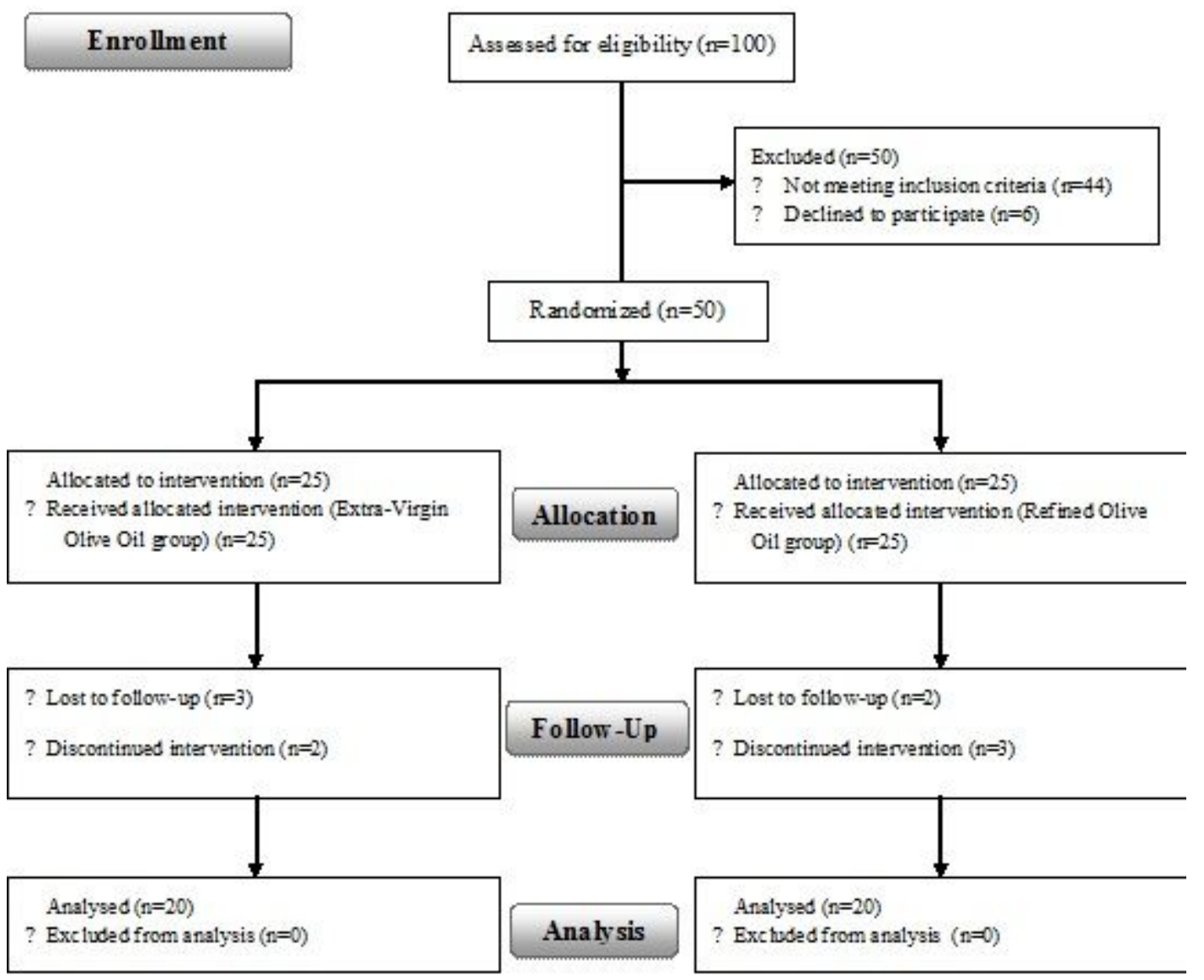

Figure l.

\section{Figure 1}

The flowchart of the study 


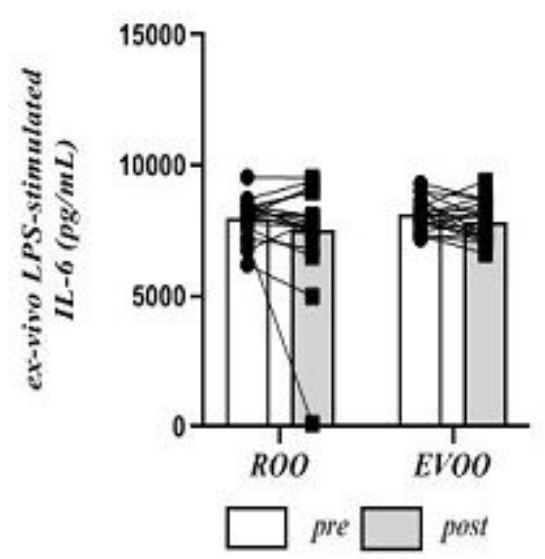

(a)

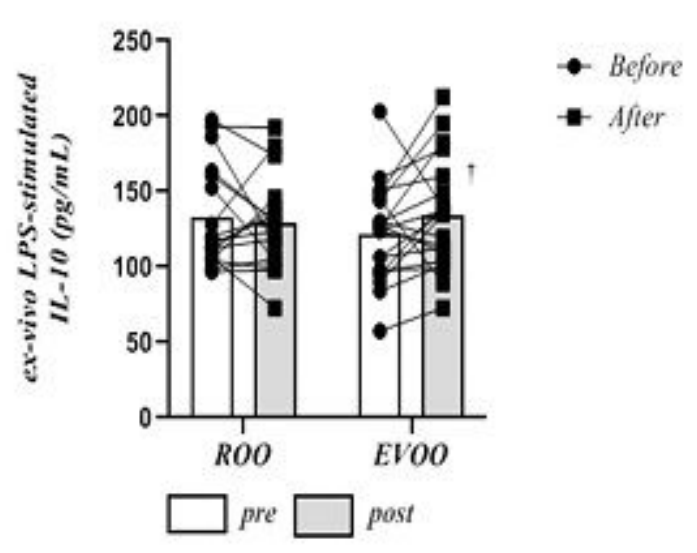

(c)

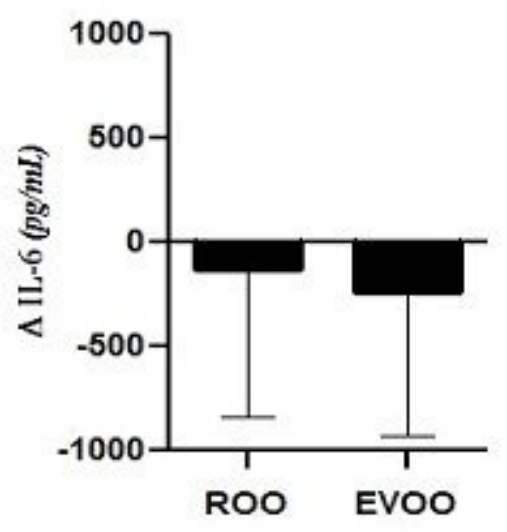

(b)

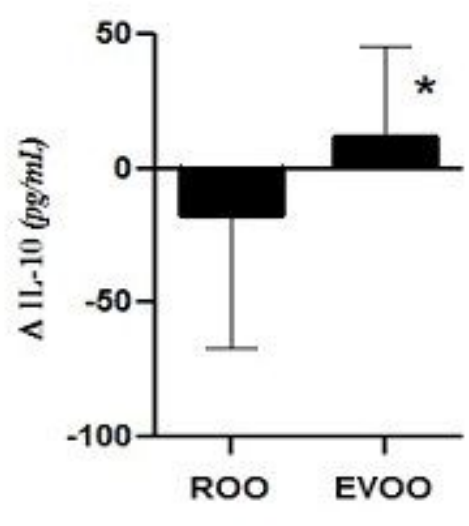

(d)

Figure 2.

Figure 2

Plasma ex-vivo whole blood LPS-stimulated IL- 6 and IL-10 values before and 6 weeks after extra virgin olive oil (EVOO) $(n=20)$ and refined olive oil $(R O O)(n=20)$ consumption. Individual pre and postresponses are represented by the lines over the bars for plasma ex-vivo IL-6 (a) and IL-10 (c). The change from baseline values $(\Delta)$ of IL- $6(\mathrm{~b})$ and IL-10 (d) are represented as well. Values are presented as Mean \pm SD. *Significantly different from ROO $(p<0.001)$. †Significantly different from baseline within the same group $(p<0.05)$.

\section{Supplementary Files}

This is a list of supplementary files associated with this preprint. Click to download. 
- CONSORT2010Checklist.doc 